\title{
Ciencia y sociedad: un nuevo reto para los profesionales de la documentación
}

\author{
Science and society: a new challenge for information professionals
}

\author{
Michela MONTESI \\ Facultad de Ciencias de la Documentación, Universidad Complutense de Madrid, España \\ mmontesi@pdi.ucm.es
}

\begin{abstract}
Resumen
Los nuevos modelos de producción de conocimiento científico vienen enfatizando desde hace un par de décadas la creciente responsabilidad de la comunidad científica ante la sociedad, tanto por lo que se refiere a las prioridades de investigación como en cuanto a los requisitos de utilidad del conocimiento generado. Sin embargo, la comunidad documental, a pesar de promover el acceso abierto a la literatura científica, sigue adhiriéndose a un concepto "elitista" de fuentes de información científica pues las concibe dentro de un sistema de comunicación científica cerrado y destinado principalmente al intercambio informativo entre pares. En este artículo, se plantean cuatro posibles vías de acercamiento entre sociedad y ciencia, proponiendo para cada de ellas un posible rol de los profesionales de la documentación. Para eso revisamos brevemente la creciente literatura sobre el acceso cada vez más importante de los ciudadanos a la información científica
\end{abstract}

Palabras clave: Fuentes de información. Sistema de comunicación científica. Sociedad. Documentalistas. Nuevos perfiles profesionales.

\section{Introducción}

Las fuentes de información científica se suelen concebir como aquellas que circulan en el sistema de la comunicación científica, en contextos profesionales específicos o que se utilizan en el ámbito de la educación superior. Los modelos tradicionales de Garvey y Griffith (1972) y Garvey (1979) proponen un sistema de comunicación científica cerrado en el cual sus principales actores, los investigadores, se consideran a la vez productores y consumidores de los productos científicos generados. Estos modelos no salen sustancialmente modificados en redefiniciones posteriores como la de Hurd (2000), que enfatiza más bien los cambios aportados por las tecnologías de la información y comunicación tanto en los canales de comunicación entre los actores de este sistema como en los medios de almacenamiento del conocimiento científico. Asimismo, estos modelos suponen la base teórica de conocidos manuales y directorios de fuentes de información como, por citar algunos

\begin{abstract}
Various models of scientific knowledge production have recently highlighted that the scientific community holds a growing responsibility towards society, both for establishing scientific priorities and for producing useful knowledge. However, the information science community, despite promoting open access to the scientific literature, keeps sticking to an elitist concept of scientific information sources, as they are conceived within a scientific communication system which is closed and intended to sustain information exchange among peers. This article analyzes four possible scenarios in which science and society may get closer, and propose for each of them a possible role for information professionals. To this end, we revise briefly the growing literature on citizens accessing scientific information.
\end{abstract}

Keywords: Information sources. Scientific communication system. Society. Information scientists. New professional profiles.

ejemplos, (Maldonado Martínez y Rodríguez Yunta, 2006) y (Cordón García, Alonso Arévalo, Gómez Díaz, \& López Lucas, 2012), o de trabajos como (Gómez Díaz, Cordón García, \& Alonso Arévalo, 2013), que emplean la expresión de fuentes especializadas, enfatizando las fuentes que circulan en un sistema de comunicación científica o profesional en cierta medida cerrado y autónomo. Contemplan, eso sí, alguna conexión con la sociedad, especialmente con el mundo de la tecnología y la empresa o introduciendo unos importantes apartados sobre las fuentes 2.0, aunque éstas se conciban principalmente desde la perspectiva de sus funcionalidades para la comunidad científica o profesional más que como herramientas de comunicación con otros actores sociales. Las propias Tecnologías de la Información y Comunicación (TIC) se pueden considerar como rompedoras con respecto a los cauces de comunicación tradicional, pero siempre dentro de la ciencia y de redes de científicos (Martínez Rodríguez, 2009). De este sistema de comunicación cientí- 
fica se ha criticado el poder que detienen las grandes editoriales en perjuicio de la comunidad académica (Sallán, Simo \& García-Parra, 2006), o se ha destacado el impacto que ha tenido en la evolución de los servicios documentales (Mulligan, 2015 y 2015a), sin poner de manifiesto su carácter cerrado con respecto a otros actores sociales. Sin embargo, la ciencia y el sistema de producción del conocimiento están cambiando y la clave del cambio parece girar alrededor de las relaciones entre ciencia y otras entidades sociales, como las empresas, los gobiernos, o la ciudadanía.

Desde la sociología de la ciencia, JiménezBuedo y Ramos Vielba (2009) revisan tres enfoques caracterizadores del sistema de producción de conocimiento actual con respecto al anterior $-\mathrm{O}$ anteriores, dependiendo del punto de vista adoptado- que se situaría antes de la crisis energética de los años setenta del siglo $\mathrm{XX}$, subrayando algunos aspectos comunes. Por un lado, la producción de conocimiento se somete al principio de la rendición de cuentas ante la sociedad. En segundo lugar, existen determinadas expectativas con respecto a la ciencia en el sentido de que debe aportar a la sociedad conocimiento útil y aplicable para la solución de problemas concretos. Como tercer aspecto remarcable del sistema actual, Jiménez-Buedo y Ramos Vielba (2009) destacan que las universidades ya no detienen el monopolio de la producción del conocimiento. Finalmente la ciencia actual se configuraría como transdisciplinar, pues es ciencia que se propone la resolución de problemas en contextos concretos y que por lo tanto requiere respuestas específicas y a la vez globales porque desarrolladas desde todos los puntos de vista posibles, tanto disciplinarios como sociales. En resumen, en este panorama cambian a la vez las modalidades de producción de conocimiento y el sistema de comunicación científica, en el sentido de que entran a formar parte de éste varios actores sociales.

El movimiento del acceso abierto a la literatura científica se sitúa en este marco, pues, entre sus objetivos prioritarios, incluye la necesidad de dar a conocer a la ciudadanía y contribuyentes lo que hacen los investigadores con el dinero público destinado a la investigación (Abadal, 2012, p. 30). Normalmente, se considera que la propia infraestructura de publicación en abierto basta para acercar la ciencia y la investigación a la sociedad (Barrionuevo Almuzara, 2009). A la hora de hablar de fuentes de información científica los profesionales de la información siguen vinculados a una concepción más cerrada de audiencia. Sin embargo, mucha investigación en el ámbito de las ciencias de la salud está poniendo de manifiesto que los ciudadanos de a pie también acceden a la literatura científica y con cada vez más interés.

Mientras la comunidad documental sigue preocupándose por la línea cada vez más borrosa entre ciencia y pseudociencia (Gray, Hamilton, Hauser, Janz, Peters \& Taggart, 2012) y alarmándose por la creciente popularidad de Google Scholar entre la comunidad científica (Chen, 2010; Hightower y Caldwell, 2012), se plantea un problema mucho más complejo cuando vemos el acceso a la literatura científica desde el punto de vista de los ciudadanos. Es muy improbable que la ciudadanía pueda penetrar en el cerradísimo sistema de la comunicación científica en los mismos términos que lo hacen los propios científicos. En una revisión sobre el impacto del acceso abierto para la ciudadanía, Zuccala (2009) concluía que la mayoría de la población no está capacitada para comprender la literatura científica, el contexto en el cual se ha producido o los mecanismos de la revisión por pares como garantía de la calidad de la información publicada. Desde este punto de vista, se configura un problema en el sentido de que las tecnologías y el acceso abierto abren la información científica al gran público y sin embargo este público no está capacitado para recibir esta información tal y como está. La comunidad documental, con todas sus especialidades, tiene una responsabilidad en esta cuestión habiendo contribuido a crear las condiciones de la publicación en abierto. ¿Qué alternativas se plantean? Y sobre todo, ¿qué papel pueden desempeñar los profesionales de la información?

\section{Objetivo, fuentes y metodología}

En este artículo, reunimos algunas propuestas e iniciativas que, tanto desde la documentación como desde otras áreas científicas, se han planteado para propiciar el acercamiento de la información científica a distintos actores sociales, aunque las alternativas, por supuesto, pueden ser mucho más numerosas. Concretamente, planteamos cuatro posibles vías de acercamiento: 1) la diseminación de la información científica y programas de divulgación en las bibliotecas públicas; 2) la producción de documentación específica para el gran público; 3) la posibilidad de que los ciudadanos actúen como colaboradores de la ciencia; y finalmente 4) la web 2.0 como espacio de interacción entre científicos y sociedad. Esta panorámica no pretende ser exhaustiva, sino propositiva con respecto a la problemática descrita arriba, y refleja un punto de vista concreto. Muchos de los ejemplos que 
proponemos provienen de las ciencias de la salud, ámbito que parece mucho más cercano que otros a la ciudadanía. Estudios anteriores han puesto de manifiesto que la ciudadanía percibe claramente las implicaciones de la investigación médica para las personas, aunque resultan menos obvias las implicaciones de la investigación en otras áreas como las ciencias químicas, físicas, o las matemáticas (Zuccala, 2010). Asimismo, los temas relativos a la salud están entre los más buscados por los ciudadanos que navegan en la web, tanto en encuestas realizadas en Estados Unidos (Cyrus, 2014) como en la Unión Europea (Kummervold y Wynn, 2012). Por lo tanto, existe claramente un interés ciudadano por temáticas científicas relacionadas con las ciencias de la salud.

La idea del trabajo surgió reuniendo una bibliografía actualizada para la asignatura de fuentes de información especializada. Con lo cual, la metodología empleada es una metodología documental, aunque el trabajo no pretende ser una revisión bibliográfica sobre el tema, sino más bien la exposición de un punto de vista argumentado a través de referencias a otros trabajos relevantes. Los trabajos se localizaron a través de la base especializada LISA y a través del buscador multidisciplinario Google Scholar, buscando "sistema de la comunicación científica" y "sociedad", o "información científica" y "sociedad", tanto en español como en inglés. También se han incorporado al artículo otros trabajos localizados siguiendo citas.

\section{Cuatro vías para acercar la información científica a la sociedad}

En lo que sigue presentamos cuatro posibles vías de acercamiento de la información científica a la sociedad, planteando asimismo posibilidades de intervención para los profesionales de la documentación. Las acciones contempladas no consisten exclusivamente en estrategias de divulgación científica, aunque están relacionadas con ésta. El concepto de "divulgación científica" implica una transmisión de conocimiento unidireccional por parte de quienes poseen conocimientos especializados hacia la sociedad. Sin embargo, en el marco de la ciencia Modo 2 o post-normal (Jiménez-Buedo y Ramos Vielba, 2009), la sociedad se contempla como un actor más en las dinámicas de producción de conocimiento científico, que además participa en la determinación de las prioridades de la investigación. Por eso se contemplan también acciones diferentes a la pura divulgación que den mayor protagonismo a la ciudadanía.

\subsection{Diseminación de la información científica} y programas de divulgación

Una posible vía para propiciar la conexión entre la información científica y la sociedad son los programas de alfabetización científica. La misión tradicional de la biblioteca ha sido la de conservar y difundir el conocimiento y la cultura. Sin embargo, en el ámbito de las bibliotecas públicas, se ha enfatizado siempre la cultura de tipo humanístico (literatura, cine...), dejando en segundo plano la cultura científica. Parece lógico que la biblioteca participe también en programas de alfabetización científica, promoviendo la cultura científica además de la humanística entre los ciudadanos, algo que se subraya también en la revisión de Zuccala (2009). En este sentido, la comunidad documental tiene un recorrido hecho puesto que desde hace unos años se vienen desarrollando proyectos de diseminación de información médica, si bien es verdad que, como muestran algunos estudios recientes, de forma aún esporádica. Luo y Park (2013), en una encuesta a nivel nacional con bibliotecarios de bibliotecas públicas estadounidenses, encontraban que los bibliotecarios americanos estaban acostumbrados a recibir consultas sobre temas de salud, especialmente sobre enfermedades específicas, aunque consideraban que carecían de la suficiente preparación en estos temas. Entre los principales obstáculos mencionaban la dificultad de interpretar las peticiones de los usuarios, la preferencia de los usuarios por las fuentes impresas a pesar de la gran abundancia de fuentes en línea, y finalmente su escasa preparación para estos servicios de asesoramiento. Luo y Park (2013) concluyen que, dada la demanda por parte de los usuarios de orientación en la información médica y teniendo en cuenta que buenos niveles de alfabetización médica suelen asociarse con un estado de salud mejor, los programas de formación en documentación médica para bibliotecarios son una necesidad. Duhon y Jameson (2013) han realizado una encuesta parecida a la anterior con el objetivo de conocer el grado de implicación de las bibliotecas universitarias americanas en programas de diseminación de información médica y recopilar experiencias al respecto. Los autores no conciben la diseminación de información médica necesariamente en términos de "alfabetización" pues entienden que las formas de participación de las bibliotecas especializadas en este reto están más bien abiertas a nuevas alternativas. En este sentido, subrayan la importancia de que las bibliotecas colaboren con otros profesionales en sus respectivas instituciones para poder ofrecer programas efectivos de diseminación científica. 


\subsection{Producción de documentación específica para el gran público}

Otra posible estrategia para acercar la información científica al gran público consiste en la producción de documentos dirigidos expresamente a éste. Según explica Björk (2007), los conocimientos científicos se van incorporando de manera paulatina a manuales o noticias de prensa, es decir a fuentes de información dirigidas al gran público. Tradicionalmente, el conocimiento científico se ha difundido entre el gran público a través de los medios de comunicación, y la mayoría de la investigación sobre divulgación de la ciencia en España trata precisamente de periodismo científico (Gonzalez Alcaide, Valderrama Zurian \& Benavent, 2009). Según datos del Observatorio de Comunicación Científica citados en Medina Aguerrebere (2012), los artículos sobre temas médicos publicados en los mayores periódicos españoles se han duplicado en la década desde 1997 a 2006, y paralelamente han crecido el número de publicaciones específicas dedicadas a estos temas, como manifestación evidente del creciente interés del público. Este mismo interés ha inducido muchas compañías privadas (farmacéuticas, de seguros, etc.) a publicar contenidos médicos en internet, con el objetivo de promocionar sus productos. A menudo estos contenidos resultan preferibles con respecto a muchos sitios web de entidades gubernamentales o sin ánimo de lucro cuya forma de ofrecer la información parece menos atractiva y sin las deseadas posibilidades de personalización (Hallyburton y Evarts, 2014). Sin embargo, una encuesta realizada con ciudadanos holandeses ha puesto de manifiesto que, en general, se tiende a percibir como más fiable la información procedente de universidades y científicos, y menos la procedente de los medios de comunicación, pues los participantes en la encuesta manifestaron la impresión de que los periodistas sean menos objetivos (Zuccala, 2010). Asimismo, en ausencia de mecanismos de colaboración con científicos e investigadores, mucha prensa divulgativa presenta imprecisiones terminológicas y de otra índole (MenesesFernández \& Martín-Gutiérrez, 2015). A todo esto cabe añadir que la formación en periodismo no suele contemplar asignaturas centradas en la comunicación científica como se suele dar en la formación en documentación. Los profesionales de la documentación, además, disponen de conocimientos técnicos en la gestión de la terminología especializada, y a menudo desempeñan sus labores en el ámbito de instituciones públicas, hecho que, teniendo en cuenta el estudio de Zuccala (2010) mencionado antes, puede conferirles mayor fiabilidad en tareas de divulgación científica propiciada desde centros institucionales, como universidades, hospitales o museos.

Algunos sugieren que los propios científicos deberían popularizar su propia investigación a través de la web (Zuccala, 2009), aunque las motivaciones de los científicos para involucrarse en actividades de divulgación parecen escasas, por lo menos en España (Torres-Albero, Fernández-Esquinas, Rey-Rocha \& Martin-Sempere, 2011). En algunas áreas, la adaptación de los contenidos científicos para el gran público cuenta con un importante camino recorrido. En la literatura médica existen documentos específicos que, con fundamento en la literatura científica, tienen el objetivo de favorecer su comprensión, su uso en la práctica clínica, o ambos. Un ejemplo muy conocido concierne a las revisiones sistemáticas publicadas por la Cochrane Library. Se trata de artículos que recopilan la última investigación disponible sobre determinadas enfermedades o intervenciones, y suelen presentar dos resúmenes, uno dirigido a los especialistas y otro, "en términos sencillos", destinado a los no especialistas y a todos los interesados, puesto que el resumen es contenido de libre acceso a través de la web. Un hecho destacado de este tipo de documento es la autoría colaborativa de la que forman parte activa los propios documentalistas, participando principalmente en la fase de búsqueda documental (Harris, 2005). No se puede excluir que los profesionales de la documentación tengan un papel en la elaboración de otras versiones más comprensibles de la investigación, y la co-autoría entre profesionales de la documentación y científicos parece la mejor estrategia para un periodismo científico de calidad (Maneses-Fernández \& Martín-Gutiérrez, 2015).

En otras áreas, como la investigación medioambiental, la comunidad científica se ve presionada por la urgencia del cambio climático para proponer conocimiento útil a la sociedad, y que, sobre todo, los agentes responsables de las políticas medioambientales puedan traducir en medidas efectivas. Dilling y Lemos (2011) subrayan que la utilidad del conocimiento depende de los distintos actores -entre los cuales están los científicos y los políticos-y que a falta de procesos iterativos de interacción entre éstos, las suposiciones de unos con respecto a lo que otros consideren útil no siempre son exactas. Los casos exitosos de uso de conocimiento científico implican o bien algún tipo de mediación, a través de brókers informativos, o bien mecanismos de coproducción del conocimiento. Por otro lado, aunque la información científica publicada en revistas con revisión por pares se percibe incluso fuera de la comunidad científica como la 
fuente de la mejor información científica disponible, muchas veces ésta no sirve para satisfacer las necesidades de conocimiento de los actores políticos (Archie, Dilling, Milford \& Pampel, 2014). Es común que a menudo la investigación realizada no sea inmediatamente útil para un problema determinado. Desde este punto de vista, la producción de documentos dirigidos a actores sociales externos a la comunidad científica parece un requisito para la aplicabilidad de los resultados de la investigación. Para los científicos implicados en FutureEarth, una plataforma para la colaboración científica internacional sobre la sostenibilidad medioambiental, no se puede abordar el problema Tierra sino desde una perspectiva múltiple (Mauser, Klepper, Rice, Schmalzbauer, Hackmann, Leemans \& Moore, 2013). Son imprescindibles tanto la integración disciplinaria como la colaboración con agentes sociales, hecho que requiere la traducción de los resultados científicos en lenguaje comprensible para todas las partes involucradas. El objetivo es ganar legitimidad, propiedad y responsabilidad de los problemas, así como opciones de resolución. En el momento en el que nos planteamos comunicar con nuevos interlocutores y se les admite en el sistema de comunicación científica, surge la necesidad de desarrollar herramientas de comunicación adecuadas, a la medida de los destinatarios finales y de un contexto específico. Quizás los profesionales de la documentación puedan tener un papel en estos procesos de mediación y traducción informativa al igual que lo tienen en otros contextos especializados, concretamente la elaboración de las revisiones sistemáticas que mencionábamos antes o en la conservación de datos, que suele darse en entornos muy especializados (Hernández-Pérez y GarcíaMoreno, 2013).

\subsection{Los ciudadanos como colaboradores de la ciencia}

Otra vía para establecer un sistema de comunicación científica más abierto en el cual se contemple la presencia de actores sociales es involucrar a estos actores en el proceso de producción de conocimiento científico. Dilling y Lemos (2011, p. 682) discuten tres opciones para aplicar el conocimiento científico a la resolución de problemas concretos: en una, la comunidad científica define los objetivos de la investigación, sin que su aplicación para la resolución de problemas sea un requisito (science push); en otra, la comunidad científica recibe unos mandatos de investigación por parte de actores sociales (demand pull), y finalmente en una tercera, y más deseable alternativa, la agenda de investi- gación se define entre productores y destinatarios en un proceso constante e iterativo de interacción. A este modelo se le denomina modelo de co-producción de conocimiento científico. Este tipo de colaboración entre comunidad científica y actores sociales está implícita asimismo en un rasgo caracterizador del sistema de producción de conocimiento actual, la transdisciplinariedad. A diferencia de la multidisciplinariedad, que sería la simple yuxtaposición de disciplinas, y de la interdisciplinariedad, que sería como la síntesis de dos o más disciplinas, la transdisciplinariedad supondría una integración previa de los supuestos epistemológicos de dos o más disciplinas para la solución de problemas de la vida real (Choi y Pak, 2006). Wickson, Carew \& Russell (2006) añaden que la investigación transdisciplinar se caracteriza, entre otros, por un concepto especial de colaboración, en la que trabajan conjuntamente los investigadores y los stakeholders de turno, es decir las personas afectadas por la propia investigación. La creciente literatura sobre la colaboración científica es una intuición de la comunidad bibliométrica con respecto a estas tendencias, reales o deseadas, de la producción de conocimiento (González Alcaide, Gómez Ferri, Agulló Calatayud, 2013). Sin embargo, en el ámbito de los estudios bibliométricos, pocas veces esta colaboración se estudia fuera del universo científico, y como mucho se establecen enlaces con el mundo de la empresa y de la tecnología (Chang, 2014). Por otra parte, en documentación, nos hemos ocupado poco de transdisciplinariedad, aunque sí que existen numerosos estudios sobre la interdisciplinariedad y la multidisciplinaried. Si buscamos los tres términos en la base de datos LISA, obtenemos 1922 resultados para interdisciplinar*, 875 para multidisciplinar $^{*}$, y tan solo 64 para transdisciplinar*. Los términos se tienden a emplear en distintas especialidades. La interdisciplinariedad parece más popular en los trabajos que estudian las prácticas de científicos e investigadores, incluidos los estudios métricos y de evaluación de la ciencia. La multidisciplinariedad, en cambio, es la característica más destacada de las grandes bases de datos Scopus, Web of Science y Google Scholar, y se trae a colación en todos los estudios que tratan de ellas. De transdisciplinariedad apenas se habla.

La biblioteca, tanto pública como especializada, podría ser un entorno prometedor para apoyar iniciativas de ciencia ciudadana y transdisciplinaria, ofreciendo un punto de encuentro entre científicos y ciudadanos, así como recursos bibliográficos, estructuras y personal cualificado. Es muy significativo que uno de los proyectos de ciencia ciudadana más conocidos, Galaxi- 
Zoo, consistiera precisamente en una labor documental, es decir en la asignación de etiquetas a millones de imágenes del espacio por parte de 150.000 colaboradores (Bonney, Phillips, Ballard et al., 2015). Los proyectos de ciencia ciudadana como GalaxiZoo que consisten en el procesamiento de datos no son los únicos, pues pueden contemplar a los ciudadanos como colaboradores en la recolección de los datos, iniciativas para el desarrollo de programas escolares, o para influir en decisiones de política local relativas a salud y medio ambiente (Bonney et al., 2015). En todos estos supuestos, la biblioteca en colaboración con otros actores sociales puede actuar de referente.

\subsection{La web 2.0 como espacio de interacción entre científicos y sociedad}

Cuando se habla en la literatura de ciencia 2.0 se hace referencia a la posibilidad de introducir en el quehacer científico las aplicaciones de la web 2.0 (Cabezas-Clavijo, Torres-Salinas \& Delgado-López-Cózar, 2009). Normalmente, la aportación de estas herramientas se concibe desde un punto de vista técnico, en términos de apoyo para las mismas operaciones comunicativas que tienen lugar offline en el sistema cerrado de la comunicación científica. Sin embargo, la web 2.0 puede proporcionar unos importantes canales de comunicación entre la comunidad científica y los distintos actores sociales, incluidos los ciudadanos. Según Zuccala (2009) el hecho de crear discusiones y debates online sobre temas científicos podría mejorar la comprensibilidad de los contenidos científicos por parte de los ciudadanos. En cuestiones de tipo médico asistimos a que los ciudadanos del mundo occidental acuden en masas a la web para buscar y compartir información relacionada con su salud. Medina Aguerrebere (2012) explica que, participando en plataformas 2.0, los ciudadanos comparten experiencias con otras personas afectas por el mismo problema y a la vez difunden contenidos médicos, ofreciendo a los profesionales de la salud oportunidades de aprendizaje e intervención. Por ejemplo, permiten hacerse una idea sobre ideas preconcebidas, aprender sobre el impacto de una patología, o detectar efectos secundarios de determinados tratamientos. Pero también permiten interactuar con los pacientes y difundir contenidos médicos apropiados. Según Marín-Torres, Aliaga, Miró, Castillo Vicente, Polentinos-Castro, \& Barral (2012), la web permite en realidad reafirmar y consolidar la relación entre pacientes y profesionales de la salud. Tanto en este estudio como en otros, como por ejemplo (Genuis, 2012), se ha comprobado que los pacientes que consultan fuentes de información en la web suelen ser más exigentes con los profesionales médicos a los que acuden, pero a la vez más responsables con su salud. Exigen además que los profesionales les remitan a fuentes de información fiables. Por supuesto la literatura médica sobre el tema es muy amplia y no es éste el lugar para revisar las posibilidades de comunicación brindadas por la web 2.0 entre la comunidad científica y la sociedad en estas áreas. Sin embargo, baste con estos ejemplos para destacar la existencia de este otro posible camino de inclusión de los actores sociales en el sistema de comunicación científica. Cabe recordar que estas posibilidades se encuentran reconocidas, aunque indirectamente, en una nueva especialidad de los estudios métricos, las altmétricas, es decir las métricas alternativas derivadas de menciones de científicos y trabajos científicos en la web. Se considera que las altmétricas podrían captar una dimensión distinta del impacto científico, como por ejemplo su impacto social (Torres Salinas, Cabezas Clavijo \& Jiménez Contreras, 2013). Sin embargo, el objetivo de la investigación altmétrica es obtener métricas válidas a efectos de la medición del impacto científico, y consecuentemente no se concibe de manera clara la web 2.0 como una oportunidad de apertura del sistema de comunicación científica. Todavía la comunidad documental no se ha planteado cómo intervenir en estas interacciones entre profesionales médicos $u$ otros científicos, y los ciudadanos a través de la web 2.0. Sin embargo, la creciente popularidad de perfiles profesionales especializados en la web social como los community managers deja entrever más posibilidades de intervención para los profesionales de la documentación en lo que es la comunicación de la información científica a los ciudadanos. La figura del community manager - que Castelló Martínez (2010, p. 78) define como "[...] aquella persona responsable de sostener las relaciones de la empresa con sus clientes en el ámbito digital, en base al conocimiento de los planteamientos estratégicos de la organización y los intereses de los clientes"se contempla en la actualidad exclusivamente para el ámbito empresarial. No obstante, en otras instituciones como hospitales, universidades, o centros de investigación, interesadas en la difusión de conocimiento científico, los community managers, adecuadamente formados, podrían ejercer labores de interacción e información con el público, promoviendo, de esta manera, el acercamiento de contenidos especializados a la ciudadanía. 


\section{Conclusiones}

En este trabajo, se ha defendido la necesidad de concebir un sistema de comunicación científica abierto en el cual tengan cabida todos los actores sociales, incluida la ciudadanía. Hemos perfilado asimismo cuatro posibles vías de acercamiento de la ciencia a la sociedad que representan a su vez nuevas oportunidades para los profesionales de la documentación: los programas de divulgación de la ciencia; la creación de documentación científica destinada al gran público; la co-producción de conocimiento científico; y finalmente, las posibilidades de interacción brindadas por la web 2.0. En cuanto a la divulgación de la ciencia, hemos visto que la biblioteca puede configurarse como un centro promotor de cultura científica y no solo humanística. Asimismo, las iniciativas de ciencia ciudadana pueden promoverse o apoyarse desde la biblioteca. Para la creación de documentación científica destinada al gran público, los profesionales de la documentación parecen los profesionales más adecuados para acompañar a los científicos, por la extensa formación en comunicación científica y terminología científica de la que disponen así como por la tradición de colaboración y participación en actividades de investigación (por ejemplo, en la elaboración de revisiones sistemáticas) y entornos altamente especializados (conservación de datos). Finalmente, se ha constatado cómo las competencias propias del community manager, un perfil profesional muy popular para los egresados en documentación, podrían emplearse para apoyar la comunicación entre ciudadanos y profesionales médicos $u$ otros investigadores en ámbitos institucionales.

En general, para los profesionales de la documentación y todas sus especialidades se abren nuevas posibilidades de mediación en entornos altamente especializados. Si tradicionalmente se ha enfatizado la perspectiva generalista de las ciencias de la documentación, las exigencias de mediación que plantea el sistema de comunicación científica actual van más bien encaminadas hacia la capacidad de traducir lo altamente técnico en conocimiento comprensible para todo el mundo, y de saber adaptarlo a circunstancias concretas.

La viabilidad de las propuestas realizadas en este artículo no se ha comprobado y representa claramente un objetivo para investigaciones futuras.

\section{Referencias}

Abadal, E. (2012). Acceso abierto a la ciencia. Barcelona: Editorial UOC.
Archie, K. M; Dilling, L; Milford, J. B.; Pampel, F. C. (2014). Unpacking the 'information barrier': Comparing perspectives on information as a barrier to climate change adaptation in the interior mountain West. // Journal of Environmental Management. 133, 397-410.

Barrionuevo Almuzara, L. (2009). Open Access: la información científica al alcance de la sociedad". // I International Meeting of Experts in Information Theories: An Interdisciplinary Approach, León (Spain, November 6th-7th 2008).

Björk, B-C. (2007). A model of scientific communication as a global distributed information system. // Information Research. 12:2, paper 307

Bonney, R; Phillips, T. B; Ballard, H. L.; Enck, J. W. (2015). Can citizen science enhance public understanding of science?. // Public Understanding of Science. 0963662515607406 .

Cabezas-Clavijo, Á; Torres-Salinas, D.; Delgado-LópezCózar, E. (2009). Ciencia 2.0: catálogo de herramientas e implicaciones para la actividad investigadora. // El Profesional de la Información. 18:1, 72-80.

Castelló Martínez, A. (2010). Una nueva figura profesional: el Community Manager. // Pangea: revista de la Red Académica Iberoamericana de Comunicación. 1, 74-97.

Chang, Y. W. (2014). Exploring scientific articles contributed by industries in Taiwan. // Scientometrics. 99:2, 599-613.

Chen, X. (2010). Google Scholar's dramatic coverage improvement. Five years after debut. // Serials Review, 36:4, pp. 221-226.

Choi, B. C.; Pak, A. W. (2006). Multidisciplinarity, interdisciplinarity and transdisciplinarity in health research, services, education and policy: 1 . Definitions, objectives, and evidence of effectiveness. Clinical and Investigative Medicine. // Medicine Clinique et Experimentale. 29:6, 351364.

Cordón García, J.A; Alonso Arévalo, J; Gómez Díaz, R; López Lucas, J. (2012). Las nuevas fuentes de información. Madrid: Ediciones Pirámide.

Cyrus, J. W. (2014). A Review of Recent Research on Internet Access, Use, and Online Health Information Seeking. Journal of Hospital Librarianship, 14:2, 149-157.

Dilling, L.; Lemos, M. C. (2011). Creating usable science: Opportunities and constraints for climate knowledge use and their implications for science policy. // Global Environmental Change. 21:2, 680-689.

Duhon, L.; Jameson, J. (2013). Health information outreach: a survey of US academic libraries, highlighting a midwestern university's experience. // Health Information \& Libraries Journal. 30:2, 121-137.

Garvey, W.D. (1979). Communication: The essence of science. Elmsford, NY: Pergamon Press.

Garvey, W.D.; Griffith, B.C. (1972). Communication and information processing within scientific disciplines: Empirical findings for psychology. // Information Storage and Retrieval. 8, 123-126.

Genuis, S. K. (2012). Constructing "sense" from evolving health information: a qualitative investigation of information seeking and sense making across sources. // Journal of the American Society for Information Science and Technology. 63:8, 1553-1566.

Gómez Díaz, R; Cordón García, J. A.; Alonso Arévalo, J. (2013). Fuentes de información especializadas de la documentación: Análisis y criterios de calidad. En Santana López, B; Travieso Rodríguez, C; Puntos de encuentro: los primeros 20 años de la Facultad de Traducción y Documentación de la Universidad de Salamanca. Salamanca: Ediciones Universidad de Salamanca. 
González Alcaide, G.; Gómez Ferri, J.; Agulló Calatayud, V. (2013). La colaboración científica: una aproximación multidisciplinar. Valencia: NauLlibres.

Gonzalez Alcaide, G; Valderrama Zurian, J. C.; Aleixandre Benavent, R. (2009). La investigación sobre la divulgación de la ciencia en España: situación actual y retos para el futuro. // Arbor. CLXXXV:738, julio-agosto, 861869

Gray, J. E; Hamilton, M. C; Hauser, A; Janz, M. M; Peters, J. P.; Taggart, F. (2012). Scholarish: Google Scholar and its value to the sciences. // Issues in Science and Technology Librarianship, 70:Summer.

Hallyburton, A.; Evarts, L. A. (2014). Gender and Online Health Information Seeking: A Five Survey MetaAnalysis. // Journal of Consumer Health On the Internet. 18:2, 128-142.

Harris MR. The librarian's role in the systematic review process: a case study. Journal of the Medical Library Association. 2005. Jan; 93:1):81-7.

Hernández-Pérez, T.; García-Moreno, M. A. (2013). Datos abiertos y repositorios de datos: nuevo reto para los bibliotecarios. El Profesional de la Información, 22:3, 259263.

Hightower, C.; Caldwell, C. (2010). Shifting sands: science researchers on Google Scholar, Web of Science, and PubMed, with implications for library collections budgets. // Issues in Science and Technology Librarianship. 63, 4.

Hurd, J. M. (2000). The transformation of scientific communication: A model for 2020. // Journal of the American Society for Information Science. 51:14, 1279-1283.

Jiménez-Buedo, M.; Ramos Vielba, I. (2009). ¿Más allá de la ciencia académica?: modo 2 , ciencia posnormal y ciencia posacadémica. // Arbor. 185:738, 721-737.

Kummervold, P. E.; Wynn, R. (2012). Health information accessed on the internet: the development in 5 European countries. // International Journal of Telemedicine and Applications. 14.

Sallán, J.M; Simo, P.; García-Parra, M. (2006). Presente y futuro del sistema de comunicación científica. // Intangible Capital. 2:2, 181-198.

Luo, L.; Park, V. T. (2013). Preparing public librarians for consumer health information service: A nationwide study. // Library \& Information Science Research. 35:4, 310-317.

Maldonado Martínez, A; Rodríguez Yunta, L. (2006, La información especializada en Internet. Madrid: Consejo Superior de Investigaciones Científicas.

Marín-Torres, V; Aliaga, J. V; Miró, I. S; del Castillo Vicente, M. I. S; Polentinos-Castro, E.; Barral, A. G. (2013). In- ternet como fuente de información sobre salud en pacientes de atención primaria y su influencia en la relación médico-paciente. // Atención Primaria. 45:1, 46-53.

Martínez Rodríguez, A. (2009). Comunicación Científica: de su necesidad a las redes y comunidades. // Bibliotecas. Anales de Investigación. V: 5, 63-67.

Mauser, W.; Klepper, G.; Rice, M.; Schmalzbauer, B. S.; Hackmann, H.; Leemans, R.; Moore, H. (2013). Transdisciplinary global change research: the co-creation of knowledge for sustainability. // Current Opinion in Environmental Sustainability. 5:3, 420-431.

Medina Aguerrebere, P.. (2012). Fuentes de información médica: Vol. 6. Barcelona: Editorial UOC

Meneses-Fernández, M. D.; Martín-Gutiérrez, J. (2015). ¿Tienen razón los investigadores al quejarse de la información periodística sobre ciencias? Experiencias con alumnos de Periodismo y científicos. // Revista Española de Documentación Científica. 38:4, e104.

Mulligan, R. (2015). The Transformation of Scholarly Communications, Part I: Context and Background. // Research Library Issues: A Report from ARL, CNI, and $\begin{array}{llll}\text { SPARC. } 287 & \text { (2015) } 2-6 . & \text { http:// publica- }\end{array}$ tions.arl.org/rli287/2.

Mulligan, R. (2015a). The Transformation of Scholarly Communications, Part II: Journal Articles and ShortForm Scholarship. // Research Library Issues: A Report from ARL, CNI, and SPARC. 287 (2015) 7-16. http://publications.arl.org/rli287/7.

Torres Salinas, D.; Cabezas Clavijo, Á.; Jiménez Contreras, E. (2013). Altmetrics: nuevos indicadores para la comunicación científica en la Web 2.0. // Comunicar. 21:41, 53-60.

Torres-Albero, C.; Fernández-Esquinas, M.; Rey-Rocha, J.; Martin-Sempere, M. J. (2011). Dissemination practices in the Spanish research system: scientists trapped in a golden cage. // Public Understanding of Science. 20:1, 12-25

Wickson, F; Carew, A. L.; Russell, A. W. (2006). Transdisciplinary research: characteristics, quandaries and quality. // Futures. 38:9, 1046-1059.

Zuccala, A. (2009). The lay person and Open Access. // Annual Review of Information Science and Technology. 43:1, 1-62.

Zuccala, A. (2010). Open access and civic scientific information literacy. // Information Research, 15:1, paper 426. http://InformationR.net/ir/15-1/paper426.html.

Enviado: 2016- 11-17. Segunda versión: 2017-02-15. Aceptado: 2017-05-09. 